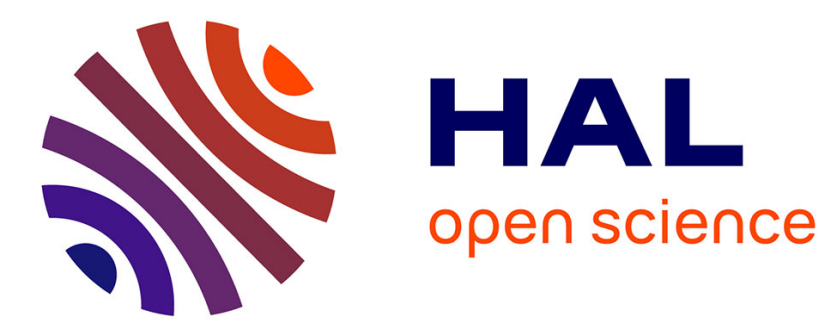

\title{
Simulating microstructure evolution during passive mixing
}

Guillaume Maitrejean, Amine Ammar, Francisco Chinesta

\section{To cite this version:}

Guillaume Maitrejean, Amine Ammar, Francisco Chinesta. Simulating microstructure evolution during passive mixing. International Journal of Material Forming, 2012, 5 (1), pp.73-81. 10.1007/s12289011-1037-8 . hal-01061439

\section{HAL Id: hal-01061439 \\ https://hal.science/hal-01061439}

Submitted on 5 Sep 2014

HAL is a multi-disciplinary open access archive for the deposit and dissemination of scientific research documents, whether they are published or not. The documents may come from teaching and research institutions in France or abroad, or from public or private research centers.
L'archive ouverte pluridisciplinaire HAL, est destinée au dépôt et à la diffusion de documents scientifiques de niveau recherche, publiés ou non, émanant des établissements d'enseignement et de recherche français ou étrangers, des laboratoires publics ou privés. 


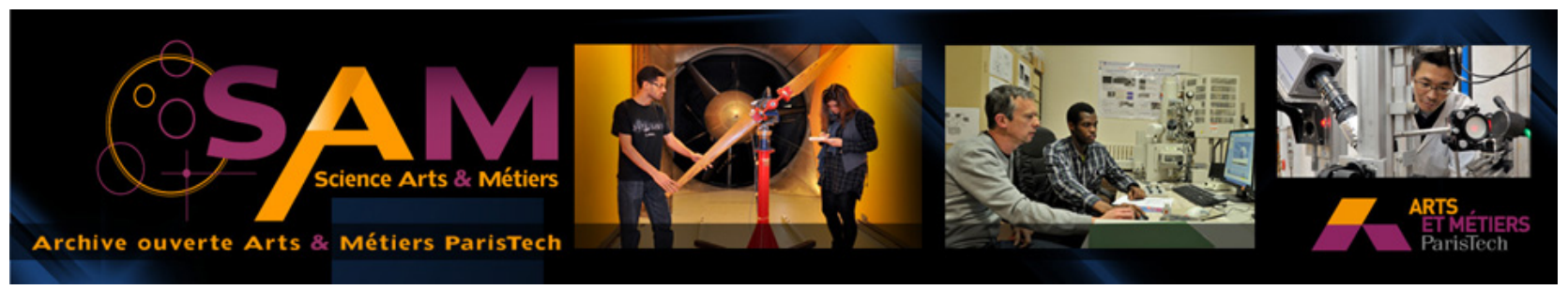

Science Arts \& Métiers (SAM)

is an open access repository that collects the work of Arts et Métiers ParisTech researchers and makes it freely available over the web where possible.

This is an author-deposited version published in: http://sam.ensam.eu

Handle ID: .http://hdl.handle.net/10985/8472

\section{To cite this version :}

Guillaume MAITREJEAN, Amine AMMAR, Francisco CHINESTA - Simulating microstructure evolution during passive mixing - International Journal of Material Forming - Vol. 5, $n^{\circ} 1$, p.73-81 2012 


\title{
Simulating microstructure evolution during passive mixing
}

\author{
Guillaume Maitrejean • Amine Ammar • \\ Francisco Chinesta
}

\begin{abstract}
The prediction of microstructure evolution during passive mixing is of major interest in order to qualify and quantify mixing devices as well as to predict the final morphology of the resulting blend. Direct numerical simulation fails because of the different characteristic lengths of the microstructure and the process itself. Micro-macro approaches could be a valuable alternative but the computational cost remains tremendous. For this reason many authors proposed the introduction of some microstructural variables able to qualify and quantify the mixing process at a mesoscale level. Some proposals considered only the effects induced by the flow kinematics, other introduced also the effects of shape relaxation due to the surface tension and coalescence. The most advanced integrate also the break-up process. However, the derivation of the evolution equations governing the evolution of such microstructural variables needs the introduction of some closure relations whose impact on the computed solution should be evaluated before applying it for simulating complex mixing flows. In this work we
\end{abstract}

G. Maitrejean $(\bowtie)$

Laboratoire de Rhéologie, UJF-INPG-CNRS,

1301 rue de la piscine, BP 53 Domaine Universitaire, 38041 Grenoble Cedex 9, France

e-mail: guillaume.maitrejean@ujf-grenoble.fr

\section{A. Ammar}

Arts et Métiers ParisTech, 2 Boulevard du Ronceray, BP 93525, 49035 Angers Cedex 01, France

e-mail: Amine.AMMAR@ensam.eu

\section{F. Chinesta}

Ecole Centrale de Nantes, 1 rue de la Noe, BP 92101 , 44321 Nantes Cedex 3, France

e-mail: Francisco.Chinesta@ec-nantes.fr consider the Lee and Park's model that considers the flow kinematics, the surface tension, the coalescence and the break-up mechanisms in the evolution of the area tensor. The accuracy of both a quadratic closure and an orthotropic relations will be analyzed in the first part of this work, and then the resulting closed model by using a quadratic closure will be used for simulating complex mixing flows.

Keywords Passive mixing - Microstructural approach • Area tensor $\cdot$ Closure relation

\section{Introduction}

When two immiscible fluids are mixed, the induced properties of the mixture are strongly dependent on the resulting microstructure, whose length scale is much smaller than the one associated with the macroscopic flow. Indeed droplets of various size and shape are formed and complex phenomena such as deformation, break-up and coalescence occur. Passive mixing implies two immiscible fluids having the same viscosity and density.

In many engineering processes, the determination of the mixing rate is very important because it is directly linked to the prediction of the mixing time and that is why the study of liquid-liquid laminar mixing has been carried out for many years and many rheological tools have been developed.

Many engineering processes benefit from good bulk mixing. In such processes, determination of mixing rate is of importance in terms of understanding and prediction of mixing time. That understanding allows defining new flows and the associated processes for improving 
the mixing rate, but one could also try to optimize other parameters related to the microstructure as for example the characteristic length, the shape and orientation of the discrete phase, ...

Mixing rates have often been quantified by a mixing time, i.e. the time required to reach a certain degree of homogeneity [11]. Most studies have based on the concentration evolution at a small number of sample points [6]. However the sensibility of the results to the number and locations of the probes is its main limitation. An alternative way of quantifying the mixing rate can be derived from the consideration of only one of the basic mechanisms of mixing processes: the increase of the material interface due to the fluid mechanics in absence of interfacial tension and molecular diffusion. The molecular diffusion leads to smooth concentration gradients across the interface. However this mechanism only becomes significant when the interface has increased significantly. It is therefore expected that the overall mixing rate will be closely linked to the rate of mechanical stretching of the interfacial area. This mechanism has been widely analyzed [10, 12]. This approach was revisited in Mackley and Neves [8] using a Lagrangian and concentration based numerical approach.

The approaches quantifying the mixing from the increase of the material interface have two important drawbacks: (i) the first one is related to the difficulty of introducing other additional physics, as the one related to the surface tension, and (ii) sometimes the microstructure description needs other information (morphology, characteristic length, shape and orientation of the discrete phase -inclusions-, ...) that the interface evolution cannot provide. The evolution of the morphology can then be modeled by predicting the change in the local morphological measure due to this velocity or deformation gradient. We will call such an approach, which treats some local characteristic morphological measure as a field variable, a micromixing analysis. From now on we focus on incompressible fluid flows and passive mixing.

The micromixing approach has been used extensively for modeling passive mixing, where interfacial energy is negligible and the two phases have identical viscosities. In passive mixing the global velocity field can be found independently of the microstructure and then used to evolve the mixture structure, described with some area tensor, very rich from the morphological and microstructural points of view. See [13] for an excellent description of this approach, in which the genesis of this kind of approaches is retraced precisely. The main difficulties noticed in that work [13] were related to the necessity of introducing a closure relation in order to derive an evolution equation for the second order area tensor. Different closure relations have been proposed, being their impact, in general, unpredictable.

A very effective way, widely used, of quantifying the mixing rate can be derived from the consideration of only one of the basic mechanisms of mixing processes: the increase of the material interface due to the flow kinematics in absence of surface tension and molecular diffusion. Thus, the higher is the interface per unit of volume, the more effective is the mixing. Because it is impossible from a practical point of view the accurate tracking of fluid interfaces, coarser descriptions have been successfully proposed.

For this purpose we can introduce the interface distribution function $\Psi(\mathbf{x}, t, \mathbf{n})$ given, at each point in the physical domain $\mathbf{x} \in \Omega$ and for any time $t$, the specific surface (i.e. interface per unit of volume) oriented in the direction $\mathbf{n}$ [5]. If we assume that the interface evolution only depends on the flow kinematics, the evolution of $\Psi(\mathbf{x}, t, \mathbf{n})$ is given by

$$
\frac{d \Psi}{d t}=-\frac{\partial}{\partial \mathbf{n}} \cdot(\dot{\mathbf{n}} \Psi)-(\nabla \mathbf{v}:(\mathbf{n} \otimes \mathbf{n})) \Psi
$$

where $\frac{d}{d t}$ denotes the material derivative, i.e. $\frac{d \Psi}{d t}=$ $\frac{\partial \Psi}{\partial t}+\mathbf{v} \cdot \nabla \Psi$, being $\mathbf{v}$ the velocity field, $\otimes$ denotes the tensor product and : the tensor product twice contracted. The time evolution of the interface orientation $\dot{\mathbf{n}}$ induced by the flow velocity field $\mathbf{v}$, is given by

$\dot{\mathbf{n}}=-(\nabla \mathbf{v})^{T} \mathbf{n}+(\nabla \mathbf{v}:(\mathbf{n} \otimes \mathbf{n})) \mathbf{n}$

On the other hand the flow kinematics induces also a variation of the interface area. Thus, a surface element $d S$ whose orientation is defined by the unit vector $\mathbf{n}$ evolves according to:

$\dot{S}=\frac{d S}{d t}=-(\nabla \mathbf{v}:(\mathbf{n} \otimes \mathbf{n})) d S$

The main difficulty related to such an approach making use of the interface distribution function $\Psi(\mathbf{x}, t, \mathbf{n})$ lies in its multidimensional character because the interface distribution function depends on the physical coordinates $\mathbf{x}$, the time, and the conformation coordinates related to the interface orientation $\mathbf{n}$.

To circumvent the curse of dimensionality related to such kinetic theory approaches coarser descriptions can be derived by introducing the so-called area tensor, that as described below contains very valuable information on the fluid morphology. Let $\Omega$ be the domain in which the flow problem is defined. Points in $\Omega$ will be referred by $\mathbf{x}$ which consists in a vector in $2 \mathrm{D}$ or $3 \mathrm{D}$. In the numerical examples presented in this work only $2 \mathrm{D}$ models are considered because in such case reference solutions can be computed. In order to quantify the 
morphology at any point $\mathbf{x} \in \Omega$ a microscopic representative volume $V(\mathbf{x})$ is considered centered at that point. This volume is small enough with respect to the macroscopic scale (related to the variation of the velocity field $\mathbf{v}$ in $\Omega$ ) but large enough with respect to the characteristic size of the microstructure. Let $S_{V}(\mathbf{x}, t)$ be the interface within $V(\mathbf{x})$ at time $t$. The area tensor $\mathbf{A}(\mathbf{x}, t)$ is then defined as:

$\mathbf{A}(\mathbf{x}, t)=\frac{1}{V(\mathbf{x})} \int_{S(\mathbf{x}, t)} \mathbf{n} \otimes \mathbf{n} d S$

The equation governing the evolution of the area tensor can be obtained by taking time derivative of Eq. 4 and then using Eqs. 2 and 3. After some manipulations it results:

$\dot{\mathbf{A}}=-(\nabla \mathbf{v})^{T} \mathbf{A}-\mathbf{A} \nabla \mathbf{v}+\nabla \mathbf{v}: \mathcal{A}$

which involves a fourth order tensor $\mathcal{A}$

$\mathcal{A}=\frac{1}{V(\mathbf{x})} \int_{S(\mathbf{x}, t)} \mathbf{n} \otimes \mathbf{n} \otimes \mathbf{n} \otimes \mathbf{n} d S$

and where the dependence of $\mathbf{A}, \mathcal{A}$ and $\mathbf{v}$ on the space and time coordinates, $\mathbf{x}$ and $t$ respectively, is omitted for the sake of clarity.

The area tensor $\mathbf{A}$ just defined is symmetric and has following appealing properties:

1. The first property concerns its trace (sum of the diagonal components of $\mathbf{A}$ ) that we symbolize by $\operatorname{Tr}(\mathbf{A})$ that taking into account the normality of $\mathbf{n}$ results:

$$
\begin{aligned}
\operatorname{Tr}(\mathbf{A}(\mathbf{x}, t)) & =\frac{1}{V(\mathbf{x})} \int_{S(\mathbf{x}, t)}\left(n_{1}^{2}+n_{2}^{2}+n_{3}^{2}\right) d S \\
& =\frac{1}{V(\mathbf{x})} \int_{S(\mathbf{x}, t)} d S=\frac{S(\mathbf{x}, t)}{V(\mathbf{x})}=S_{V}(\mathbf{x}, t)
\end{aligned}
$$

Thus, the trace of the area tensor gives the specific surface whose maximization is currently searched in mixing processes.

2. If we define the volume fraction of the disperse phase as $\phi$, assumed uniformly distributed within $\Omega$, then we can define a characteristic length $L(\mathbf{x}, t)$ of the microstructure at point $\mathbf{x}$ from the volume of the discrete phase $V_{d}(\mathbf{x})=\phi \cdot V(\mathbf{x})$ :

$L(\mathbf{x}, t)=\frac{V_{d}(\mathbf{x})}{S(\mathbf{x}, t)}=\frac{\phi \cdot V(\mathbf{x})}{S(\mathbf{x}, t)}=\frac{\phi}{S_{V}(\mathbf{x}, t)}=\frac{\phi}{\operatorname{Tr}(\mathbf{A}(\mathbf{x}, t))}$

3. In passive mixing the strain rate varies linearly at the scale of $V(\mathbf{x})$, so that an initially spherical discrete domain will deform into an ellipsoid for any state of strain. This characteristic ellipsoid provides a convenient way to interpret the area tensor. The microstructure shape and orientation can be easily deduced from the normalized area tensor $\tilde{\mathbf{A}}$ defined as

$\tilde{\mathbf{A}}(\mathbf{x}, t)=\frac{\mathbf{A}(\mathbf{x}, t)}{\operatorname{Tr}(\mathbf{A}(\mathbf{x}, t))}$

and whose eigenvalues allow computing the length of the ellipsoid axes, being their orientation given by the associated eigenvectors. See [13] for more details concerning the relation between that ellipsoid and the area tensor.

It is easy to verify that the interface distribution function and the area tensor descriptions can be linked by taking into account the expression

$\mathbf{A}(\mathbf{x}, t)=\int_{\mathcal{B}}(\mathbf{n} \otimes \mathbf{n}) \Psi(\mathbf{x}, t, \mathbf{n}) d \mathbf{n}$

where $\mathcal{B}$ represents the surface of the unit sphere.

The solution of Eq. 5 requires the introduction of a closure relation for expressing the fourth order area tensor $\mathcal{A}$ as a function of the second order one $\mathbf{A}$. Different closure relations have been proposed, being the simplest ones, the quadratic and the orthotropic [13].

The quadratic closure, initially proposed by Doi and Ohta, writes:

$\mathcal{A}=\frac{1}{S_{V}}(\mathbf{A} \otimes \mathbf{A})$

The impact of the quadratic closure relation was deeply analyzed in [3] where the solution computed by using Eq. 5 with the quadratic closure (Eq. 11) was compared to the area tensor obtained from the interface distribution function $\Psi(\mathbf{x}, t, \mathbf{n})$ according to Eq. 10, where the distribution function $\Psi(\mathbf{x}, t, \mathbf{n})$ was computed by integrating Eq. 1 without the necessity of introducing any closure.

The orthotropic relation proposed by [4] postulate a different dependence of $\mathcal{A}$ on $\mathbf{A}$ [13].

Until now, the only mechanism affecting the interface evolution is the flow kinematics. However one could expect that other phenomena could participate actively in the interface evolution, as for example the surface tension, the droplets coalescence or the droplets break-up.

\section{Enriched modeling}

One could expect that in absence of flow the microstructure evolves toward an isotropic state, i.e. 
spherical droplets, and that the interface area decreases because the droplets coalescence. These mechanisms are expected to be dependent on the matrix viscosity $\eta$ and the surface tension $\Gamma$.

Thus, Doi and Ohta considered the evolution of the specific surface, i.e. the trace of the area tensor, given by

$$
\dot{S}_{V}=-r_{1} \cdot S_{V}
$$

whereas the deviatoric part of the area tensor tends to vanish in absence of flow

$\frac{d}{d t}\left(\frac{\mathbf{A}-\frac{T r(\mathbf{A})}{3} \mathbf{I}}{S_{V}}\right)=-r_{2} \cdot\left(\frac{\mathbf{A}-\frac{T r(\mathbf{A})}{3} \mathbf{I}}{S_{V}}\right)$

where $r_{1}$ and $r_{2}$ represents two relaxation rates.

A dimensional analysis leads to:

$$
\begin{aligned}
& r_{1}=c_{1} \frac{\Gamma \cdot S_{V}}{\eta} \\
& r_{2}=c_{2} \frac{\Gamma \cdot S_{V}}{\eta}
\end{aligned}
$$

where $c_{1}$ and $c_{2}$ are two positive dimensionless coefficients.

Lee and Park considered a third mechanism related to the droplet break-up whose effect on the evolution of the specific surface was assumed depending on the deviatoric part of the area tensor according to [7]:

$$
\dot{S}_{V}=-c_{3} \frac{\Gamma}{\eta}\left(\left(\mathbf{A}-\frac{\operatorname{Tr}(\mathbf{A})}{3} \mathbf{I}\right):\left(\mathbf{A}-\frac{\operatorname{Tr}(\mathbf{A})}{3} \mathbf{I}\right)\right)
$$

Thus the effects of these extra-mechanics write:

$$
\begin{aligned}
& \left.\frac{d}{d t} S_{V}\right|_{\text {extra }}=-c_{1} \frac{\Gamma S_{V}^{2}}{\eta}-c_{3} \frac{\Gamma}{\eta}\left(\mathbf{A}-\frac{\operatorname{Tr}(\mathbf{A})}{3} \mathbf{I}\right) \\
& :\left(\mathbf{A}-\frac{\operatorname{Tr}(\mathbf{A})}{3} \mathbf{I}\right)
\end{aligned}
$$

and

$$
\left.\frac{d}{d t}\left(\frac{\mathbf{A}-\frac{T r(\mathbf{A})}{3} \mathbf{I}}{S_{V}}\right)\right|_{\text {extra }}=-c_{2} \frac{\Gamma S_{V}}{\eta}\left(\frac{\mathbf{A}-\frac{T r(\mathbf{A})}{3} \mathbf{I}}{S_{V}}\right)
$$

where $c_{1}, c_{2}$ and $c_{3}$ are three positive dimensionless coefficients which could depend on the volume fraction $\phi$.

By introducing the new dimensionless coefficients $\lambda=c_{1}+c_{2}, \mu=c_{1} /\left(c_{1}+c_{2}\right)$ and $v=c_{3} /\left(c_{1}+c_{2}\right)$, the equation governing the evolution of the area tensor writes:

$$
\begin{aligned}
\frac{d \mathbf{A}}{d t}= & -(\nabla \mathbf{v})^{T} \mathbf{A}-\mathbf{A} \nabla \mathbf{v}+\nabla \mathbf{v}: \mathcal{A} \\
& -\lambda \frac{\Gamma}{\eta} S_{V}\left(\mathbf{A}-\frac{S_{V}}{3} \mathbf{I}\right)-\lambda \mu \frac{\Gamma}{\eta} S_{V}{ }^{2} \frac{\mathbf{I}}{3} \\
& -\lambda \nu \frac{\Gamma}{\eta}\left(\mathbf{A}: \mathbf{A}-\frac{S_{V}{ }^{2}}{3}\right) \frac{\mathbf{A}}{S_{V}}
\end{aligned}
$$

\section{Evaluating the impact of closure relations}

Chinesta and Mackley [3] analyzed the impact of the quadratic closure in the evolution of the area tensor in absence of droplets break-up. In this section we are performing a similar analysis when shape relaxation, coalescence and break-up mechanisms are present.

Inspired from the procedure described in [3], it is easy to prove that the equation governing the evolution of the interface distribution function reads:

$$
\begin{aligned}
\frac{d \Psi}{d t}= & -\frac{\partial}{\partial \mathbf{n}}(\dot{\mathbf{n}} \Psi)-(\nabla \mathbf{v}:(\mathbf{n} \otimes \mathbf{n})) \Psi \\
& -\frac{\partial}{\partial \mathbf{n}}\left(D(\Psi) \frac{\partial \Psi}{\partial \mathbf{n}}\right)+F(\Psi)
\end{aligned}
$$

where the diffusion coefficient and the source terms are given by:

$D(\Psi)=\lambda \frac{\Gamma}{\eta} \frac{1}{6}\left(S_{V}+\frac{v}{S_{V}}\left(\mathbf{A}: \mathbf{A}-\frac{S_{V}^{2}}{3}\right)\right)$

and

$F(\Psi)=-\lambda \frac{\Gamma}{\eta} \frac{1}{4 \pi}\left(\mu S_{V}^{2}+v\left(\mathbf{A}: \mathbf{A}-\frac{S_{V}^{2}}{3}\right)\right)$.

with

$\mathbf{A}=\int_{\mathcal{B}}(\mathbf{n} \otimes \mathbf{n}) \Psi d \mathbf{n}$

The solution of Eqs. 20-23 allows computing the interface distribution function, and then the second order and the fourth order area tensor, $\mathbf{A}$ and $\mathcal{A}$ respectively, without the introduction of any closure relation. The price to be paid is the solution of a model defined in a multidimensional space. In general complex flows the solution of these equations is quite difficult despite the recent progresses accomplished in numerical strategies able to circumvent or at least alleviate the multidimensionality issue $[1,2,9]$. 
In the case of simple rheometric flows we can assume a homogeneous kinematics and then ignore the dependence of all the variables on the physical coordinates. In this case, the resulting kinetic theory model making use of the interface distribution function can be solved easily because it only needs an appropriate discretization of the surface of the unit sphere.

\section{Numerical results}

Area tensor evolution equation: Dimensionless form

The area tensor equation can be rewritten in the dimensionless form by introducing the dimensionless capillary number $\mathrm{Ca}$ defined by

$C a=\frac{\eta \dot{\gamma}}{\Gamma S_{V}^{\text {init }}}$

where $\eta$ is the viscosity, $S_{V}^{\text {init }}$ the initial specific surface, $\Gamma$ the surface tension and $\dot{\gamma}$ the equivalent strain rate defined by $\dot{\gamma}=\sqrt{\nabla \mathbf{v}: \nabla \mathbf{v}}$.

The dimensionless form is obtained by affecting both the orientation tensor and the specific surface by the initial specific surface and time by the inverse of the shear rate. Thus, the dimensionless form of the equation governing the area tensor evolution reads

$$
\begin{aligned}
\frac{d \mathbf{A}}{d t}= & -(\nabla \mathbf{v})^{T} \mathbf{A}-\mathbf{A} \nabla \mathbf{v}+\nabla \mathbf{v}: \mathcal{A} \\
& +\left(-\lambda \mu \frac{1}{C a} S_{V}^{2}\right) \frac{\mathbf{I}}{3}-\lambda \frac{1}{C a} S_{V}\left(\mathbf{A}-\frac{S_{V}}{3} \mathbf{I}\right) \\
& -\lambda v \frac{1}{C a}\left(\mathbf{A}: \mathbf{A}-\frac{S_{V}^{2}}{3}\right) \frac{\mathbf{A}}{S_{V}}
\end{aligned}
$$

Evaluating the closure relations in a simple shear flow

In what follows we analyzed the evolution of the specific surface $S_{V}$ for two different capillary numbers $C a=1$ and $C a=10$ in the simple shear and bielongational flows defined respectively by:

$$
\nabla \mathbf{v}=\left(\begin{array}{ccc}
0 & 1 & 0 \\
0 & 0 & 0 \\
0 & 0 & 0
\end{array}\right)
$$

and

$$
\nabla \mathbf{v}=\left(\begin{array}{ccc}
-1 & 0 & 0 \\
0 & 0.5 & 0 \\
0 & 0 & 0.5
\end{array}\right)
$$

Different values of the parameters $\lambda, \mu$ and $v$ where considered for analyzing their effect on the time evolution of the specific area.

Figures 1 and 2 depict the specific surface time evolution in the simple shear flow for $C a=1$ and $C a=10$ respectively, whereas Figs. 3 and 4 show similar results in the case of the bi-elongational flow. In these figures we can appreciate the impact of the closure relations. Despite the fact that the quadratic closure relation introduces in some cases an appreciable deviation with respect to the reference solution computed within the kinetic theory framework, its implementation is the most efficient from the computational time viewpoint. In these figures we can also notice the strong sensibility of the solution to the model parameters $C a, \lambda, \mu$ and $\nu$.

\section{Simulating complex flows}

We consider the mixing device whose geometry is depicted in Fig. 5. The area tensor will be represented by using the ellipse associated to the rotation of $\frac{\pi}{2}$ of tensor A. Such representation is closer to the real microstructure morphology. The ellipse axes are given by the eigenvectors of tensor $\mathbf{A}$ rotated of $\frac{\pi}{2}$ whereas their lengths are related to the associated eigenvalues. We are also representing using a color map the value of the inverse of the specific surface that is a key parameter for quantifying the mixing. Initially, the droplets should be represented as spheres, i.e. area tensor is diagonal, all the components having the same value.

In the simulations that we address in this section we consider a coupling between the flow kinematics and the microstructure evolution:

- Flow kinematics

$$
\begin{aligned}
\nabla \cdot \sigma & =0 \\
\nabla \cdot \mathbf{v} & =0 \\
\sigma & =-p \mathbf{I}+2 \eta \mathbf{D}-\frac{\Gamma}{\eta}\left(\mathbf{A}-S_{V} \frac{\mathbf{I}}{3}\right)
\end{aligned}
$$

- Microstructure evolution

$$
\begin{aligned}
\frac{d \mathbf{A}}{d t}= & -(\nabla \mathbf{v})^{T} \mathbf{A}-\mathbf{A} \nabla \mathbf{v}+\nabla \mathbf{v}: \mathcal{A} \\
& +\left(-\lambda \mu \frac{1}{C a} S_{V}^{2}\right) \frac{\mathbf{I}}{3}-\lambda \frac{1}{C a} S_{V}\left(\mathbf{A}-\frac{S_{V}}{3} \mathbf{I}\right) \\
& -\lambda \nu \frac{1}{C a}\left(\mathbf{A}: \mathbf{A}-\frac{S_{V}^{2}}{3}\right) \frac{\mathbf{A}}{S_{V}}
\end{aligned}
$$

where a quadratic closure relation was considered, because it represents a good compromise between accuracy and computational efficiency. 


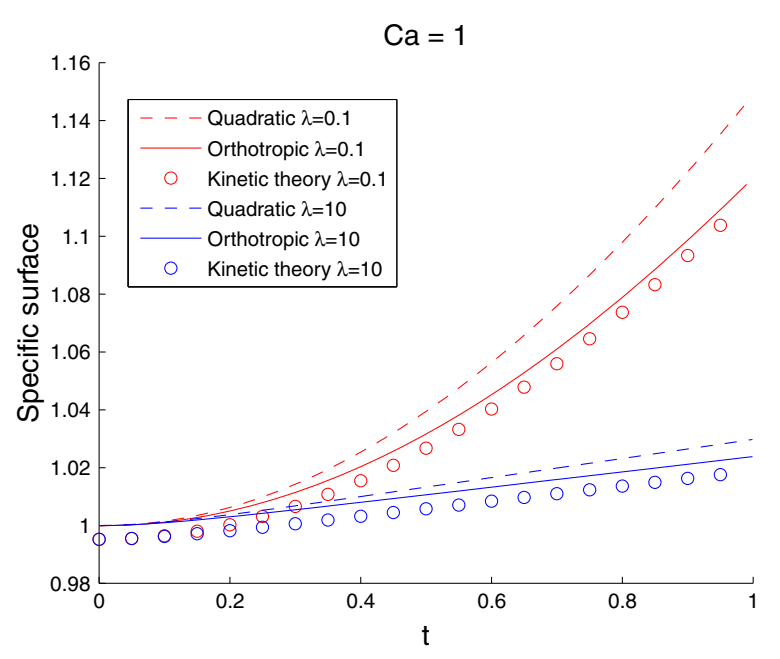

(a) $\lambda=0.1$ (red) and $\lambda=10$ (blue)

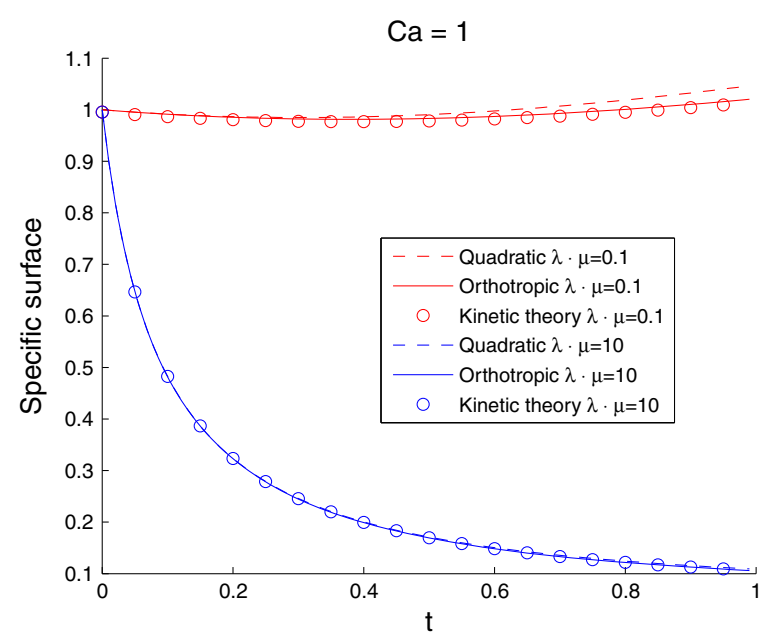

(b) $\lambda \cdot v=0.1$ (red) and $\lambda \cdot v=10$ (blue)

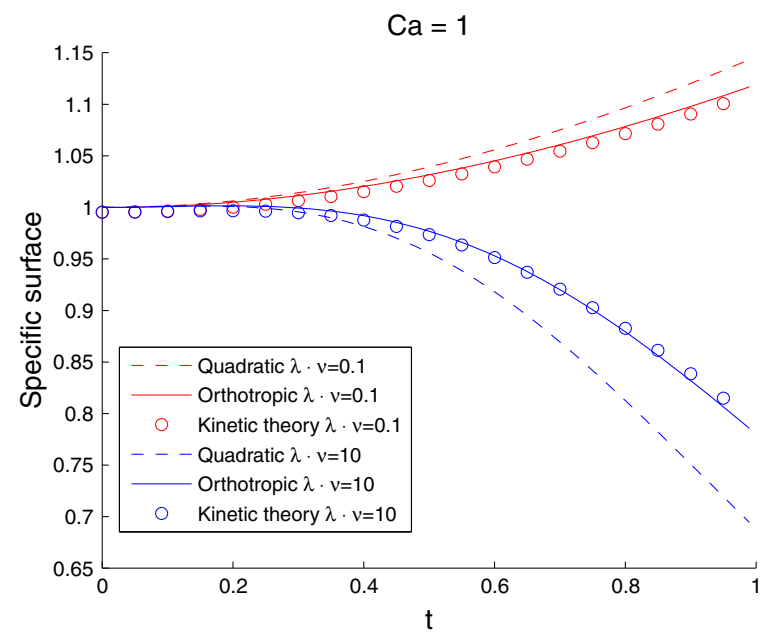

(c) $\lambda \cdot \mu=0.1$ (red) and $\lambda \cdot \mu=10$ (blue)

Fig. 1 Specific surface evolution in the simple shear flow with $C a=1$

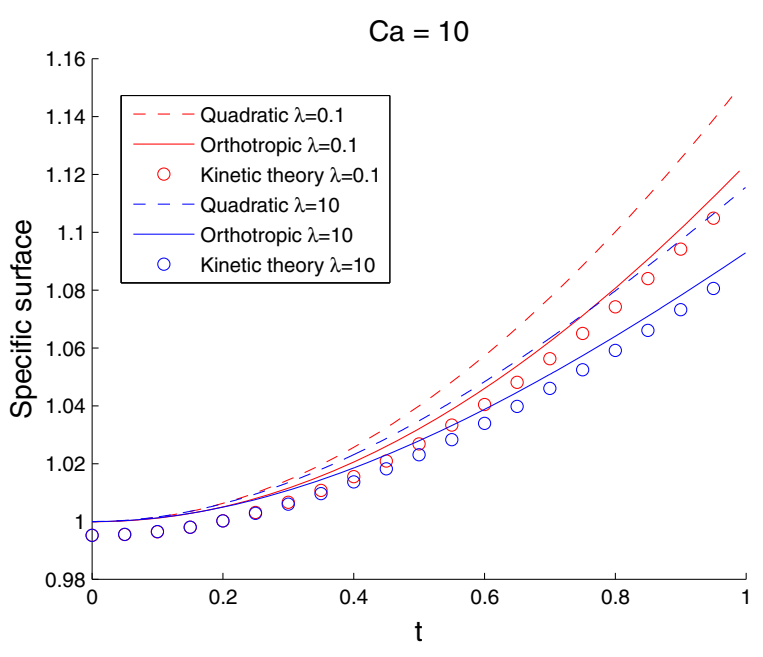

(a) $\lambda=0.1$ (red) and $\lambda=10$ (blue)

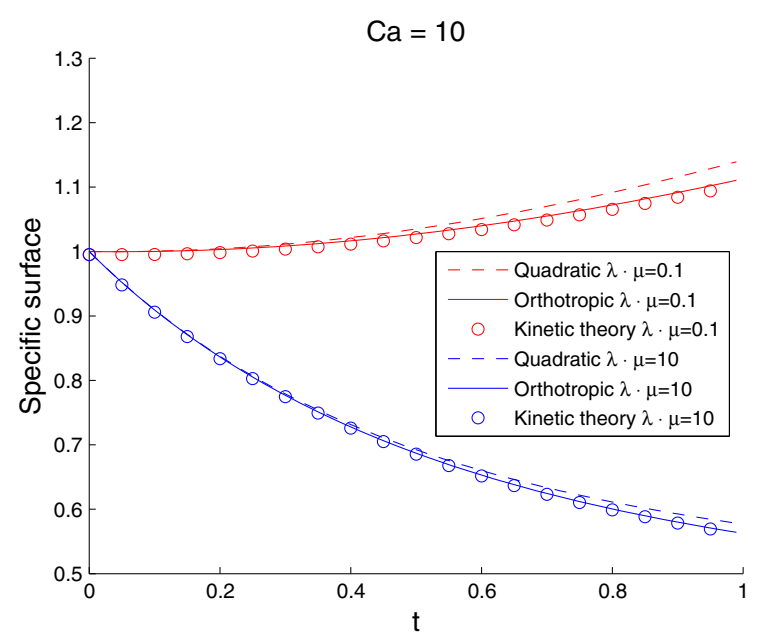

(b) $\lambda \cdot v=0.1$ (red) and $\lambda \cdot v=10$ (blue)

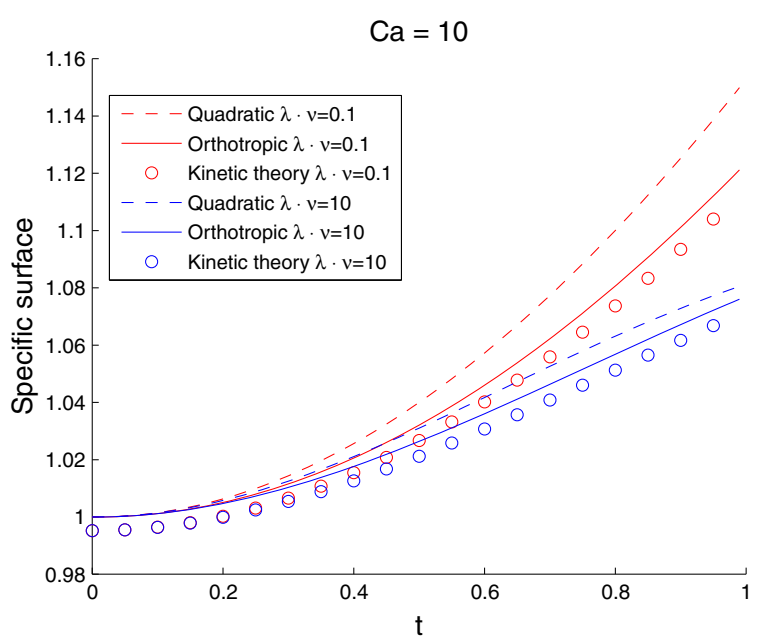

(c) $\lambda \cdot \mu=0.1$ (red) and $\lambda \cdot \mu=10$ (blue)

Fig. 2 Specific surface evolution in the simple shear flow with $\mathrm{Ca}=10$ 


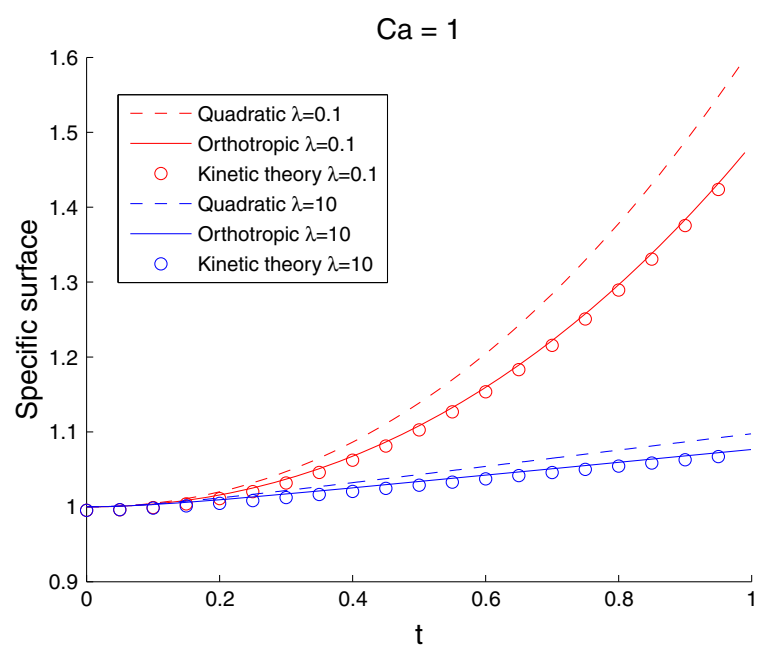

(a) $\lambda=0.1$ (red) and $\lambda=10$ (blue)

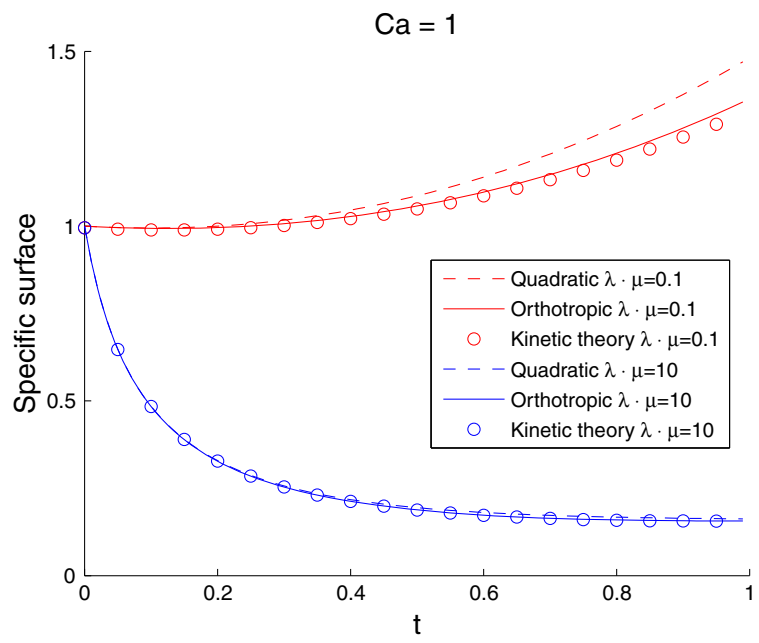

(b) $\lambda \cdot v=0.1$ (red) and $\lambda \cdot v=10$ (blue)

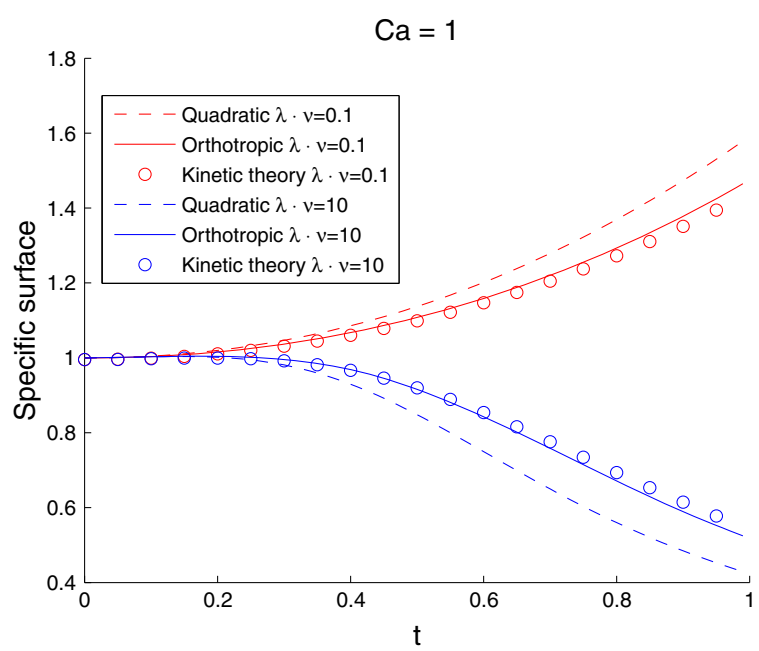

(c) $\lambda \cdot \mu=0.1$ (red) and $\lambda \cdot \mu=10$ (blue)

Fig. 3 Specific surface evolution in the bi-elongational flow with $C a=1$

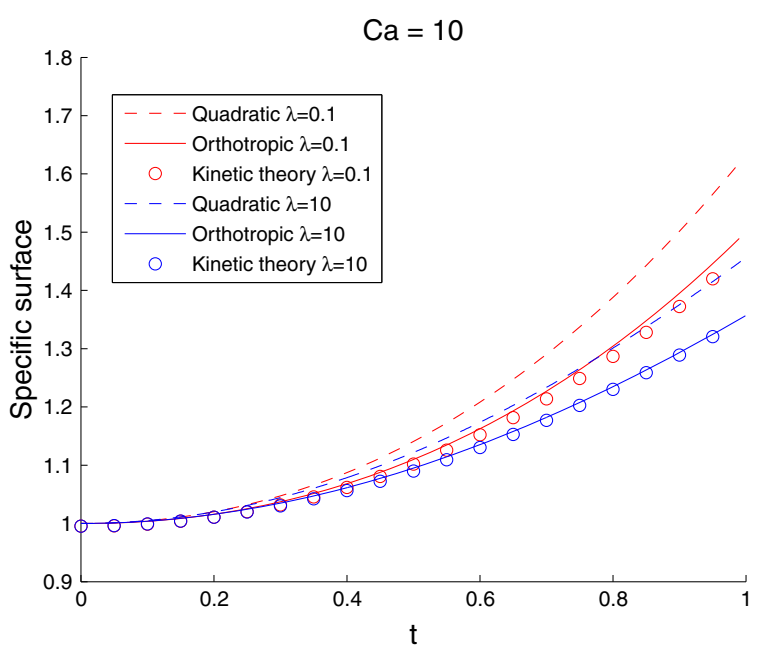

(a) $\lambda=0.1$ (red) and $\lambda=10$ (blue)

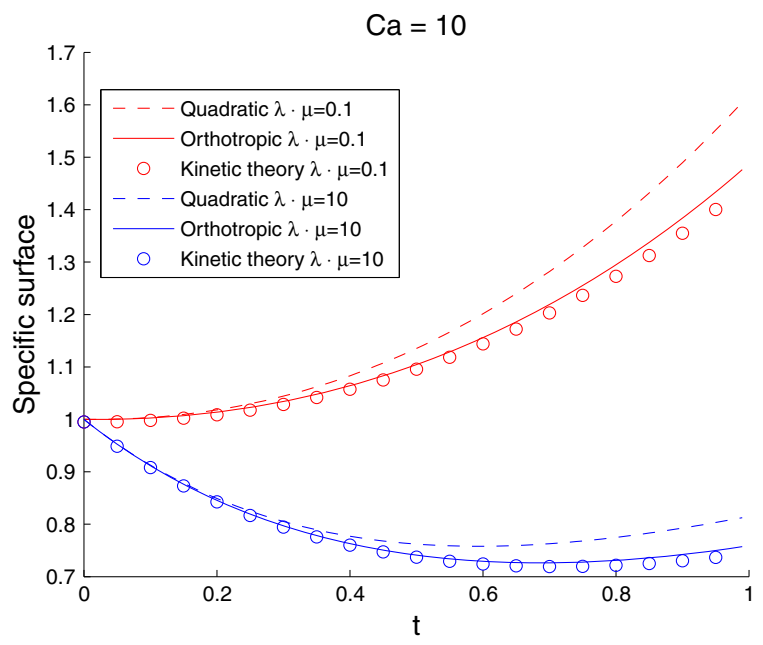

(b) $\lambda \cdot v=0.1$ (red) and $\lambda \cdot v=10$ (blue)

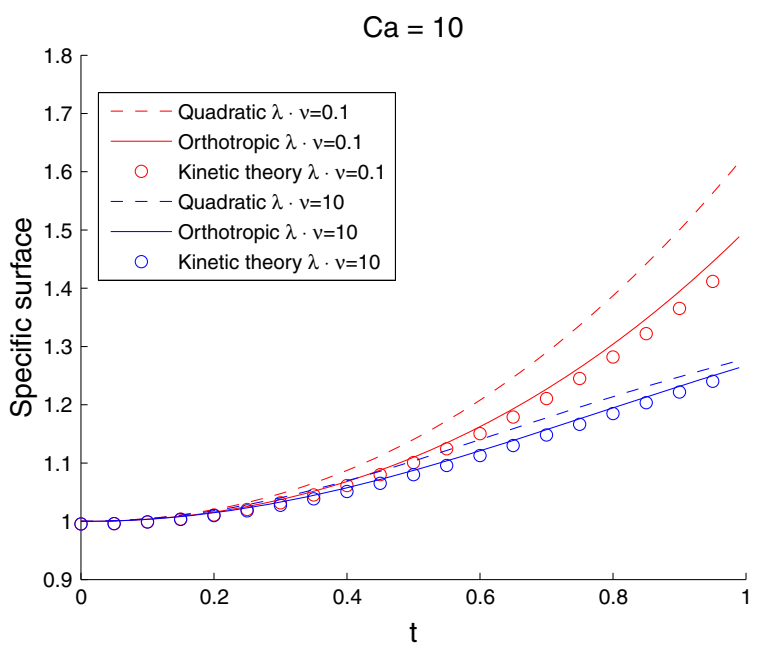

(c) $\lambda \cdot \mu=0.1$ (red) and $\lambda \cdot \mu=10$ (blue)

Fig. 4 Specific surface evolution in the bi-elongational with $C a=10$ 


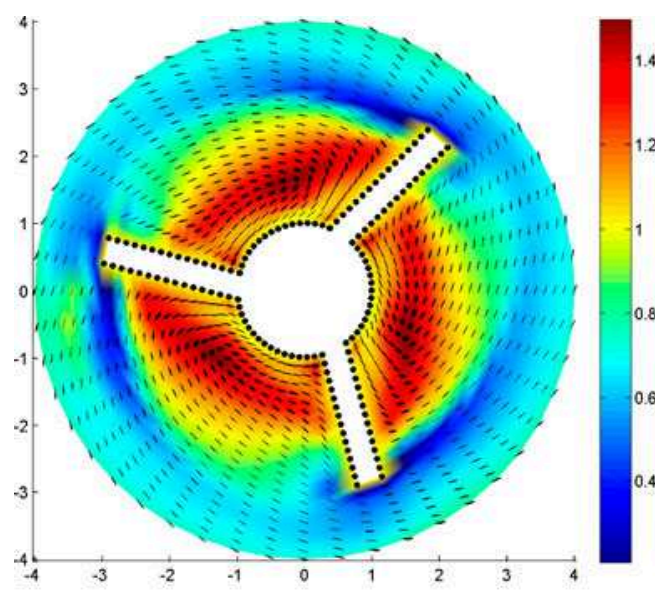

(a) $\theta=\frac{2 \pi}{3}$

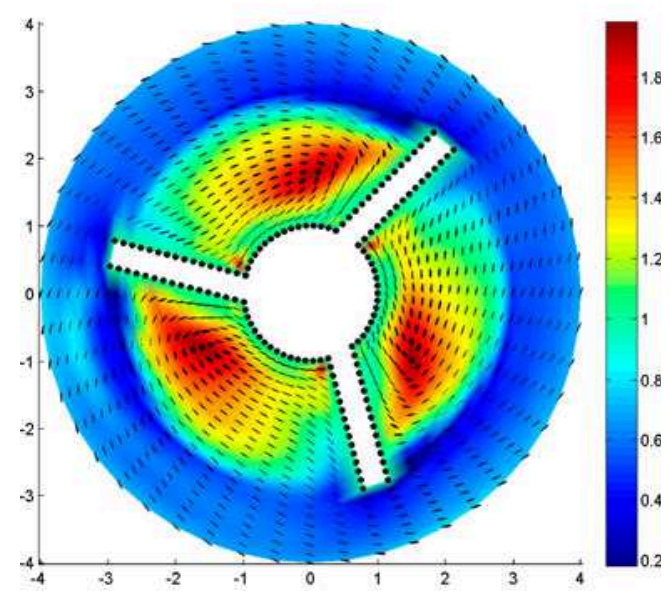

(b) $\theta=\frac{4 \pi}{3}$

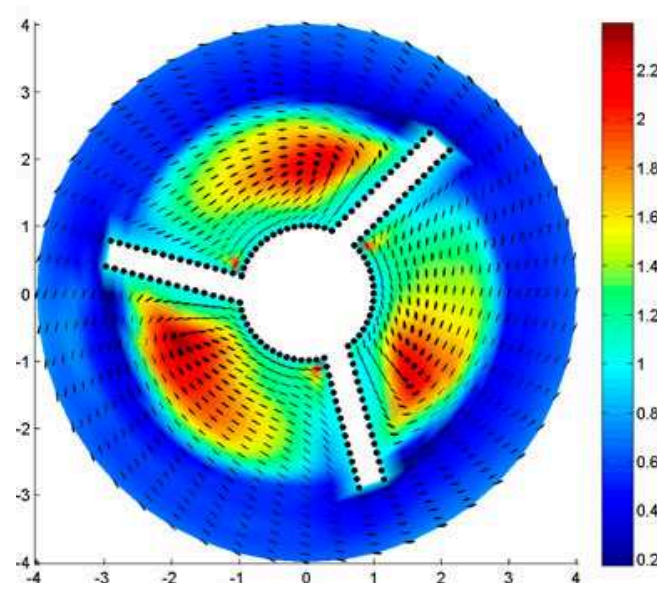

(c) $\theta=2 \pi$

Fig. 5 Area tensor evolution during a complete turn of the rotor

In the simulations we consider the following choice of the model parameters: $\lambda=0.1, \mu=0.1$ and $v=0.15$ and $C a=1$. Figure 5 illustrates the microstructure evo-

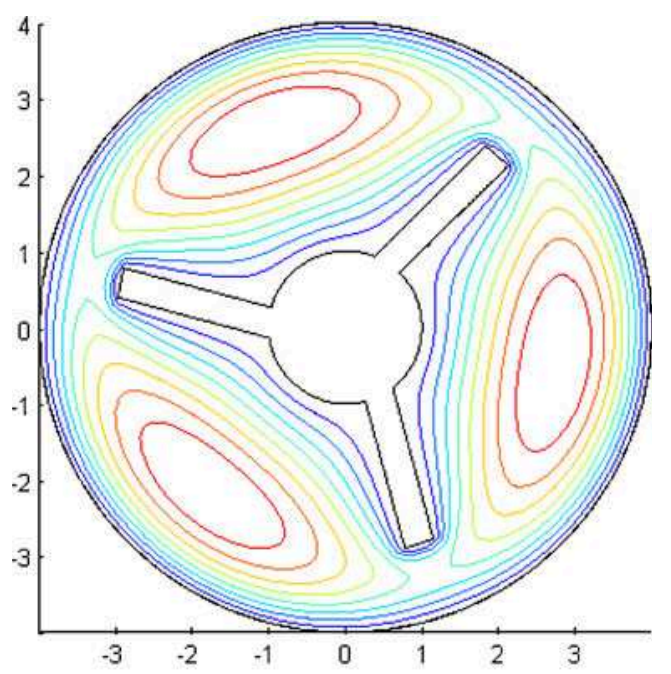

(a) Streamlines for a isotropic microstructure

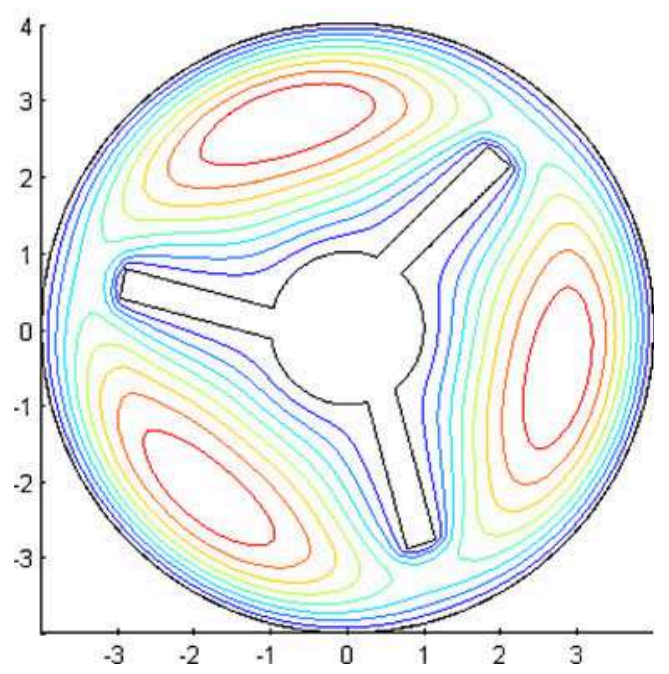

(b) Streamlines after a complete turn

Fig. 6 Influence of the microstructure evolution on the flow streamlines

lution during a complete turn of the rotor as well as the evolution of the specific surface.

Finally, Fig. 6 compares the streamlines for two different microstructural morphologies: the initial one perfectly isotropic and the one induced after a complete turn. It can be noticed that in the flow here considered the difference is not too significative.

\section{Conclusion}

The use of the area tensor allows describing the morphology evolution during mixing processes at the mi- 
croscopic scale and is very convenient in term of microstructural description (specific interface, shape, orientation, ....).

In this paper we considered a model taking into account the flow effects, surface tension, droplets coalescence and droplets break-up. The equation governing the evolution of the area tensor in that model involves a fourth order area tensor that must be written as a function of the second order orientation tensor by using an appropriate closure relation that introduces in general an unpredictable error. To evaluate the effects of a quadratic or an orthotropic closure relation in the area tensor evolution first we derive a kinetic theory model governing the evolution of the so-called interface distribution function that does not involve any closure relation, from which the different area tensors can be calculated. Then, we compared in a simple shear flow the reference solution (computed from the interface distribution function) and the ones obtained by using both closure relations. Both closure relations seem to be good, and the quadratic one was retained because its simplicity. Finally, the model was used for simulating complex flows in mixing devices.

\section{References}

1. Ammar A, Mokdad B, Chinesta F, Keunings R (2006) A new family of solvers for some classes of multidimensional partial differential equations encountered in kinetic theory modeling of complex fluids. J Non-Newton Fluid Mech. 139: $153-176$
2. Ammar A, Mokdad B, Chinesta F, Keunings R (2007) A new family of solvers for some classes of multidimensional partial differential equations encountered in kinetic theory modeling of complex fluids. Part II: transient simulation using spacetime separated representations. J Non-Newton Fluid Mech 144:98-121

3. Chinesta F, Mackley MR (2008) Microstructure evolution during liquid-liquid laminar mixing: a kinetic theory approach. Int J Mater Form 1:47-55

4. Cintra JS, Tucker Ch L (1995) Orthotropic closure approximations for flow-induced fiber orientation. J Rheol 39:10951122

5. Doi M, Ohta T (1991) Dynamics and rheology of complex interfaces. i. J Chem Phys 95:1242-1248

6. Hall KR, Godfrey JC (1968) The mixing rates of highly viscous Newtonian and non-Newtonian fluids in a laboratory sigma-blade mixer. Trans Inst Chem Eng 46:205-212

7. Lee HM, Park OO (1994) Rheology and dynamics of immiscible polymer blends. J Rheol 38:1405-1425

8. Mackley MR, Neves Saraiva RMC (1999) The quantitative description of fluid mixing using Lagrangian- and concentration-based numerical approaches. Chem Eng Sci 54:159-170

9. Mokdad B, Ammar A, Normandin M, Chinesta F, Clermont JR (2010) A fully deterministic micro-macro simulation of complex flows involving reversible network fluid models. Math Comput Simul 80:1936-1961

10. Ottino JM (1989) The kinematics of mixing: stretching, chaos and transport. Cambridge University Press

11. Rielly CD, Smith DLO, Lindley JA, Niranjan K, Philips VR (1994) Mixing processes for agricultural and food materials: Part 4, assessment and monitoring of mixing systems. J Agric Eng Res 29:1-18

12. Roberts EPR, Mackley MR (1995) The simulation of stretch rates for the quantitative prediction and mapping of mixing within a channel flow. Chem Eng Sci 50:3727-3746

13. Wetzel ED, Tucker ChL (1999) Area tensors for modeling microstructure during laminar liquid-liquid mixing. Int J Multiph Flow 25:35-61 